chrom-P450- Enzyme (CYP-Enzyme) beeinflussen [3]. Vor allem eine Hemmung der CYP3A4 und CYP2C9 könnte durch langsameren Abbau der auf diesem Weg metabolisierten Medikamente die Gefahr von Nebenwirkungen erhöhen. Speziell CYP3A4 ist in die Verstoffwechslung zahlreicher klassischer und neuer Zytostatika und Immunsuppressiva involviert.

\section{Keine Hinweise auf relevante Interaktionen mit antitumoralen Therapien}

Trotz dieser präklinischen Hinweise auf CYP-Hemmung durch Inhaltsstoffe von Ingwer fehlt die Evidenz für eine klinische Relevanz dieser Beobachtungen. Zudem beziehen sich die Daten auf Ingwerpräparate, nicht auf die frische Knolle. Weitere Studien sind gefordert, die auch Aufschluss darüber geben, wie stark die mutmaßliche CYP-Hemmung ist. Die vorliegenden Ergebnisse sollten aber auf jeden Fall Patienten dafür sensibilisieren, den be- handelnden Ärzten die Einnahme oder den Verzehr von Ingwer in größeren Mengen zu berichten. Dies gilt im besonderen Maße bei zeitgleicher Anwendung von neuen Medikamenten, über deren Wechselwirkungspotenzial man noch nicht viel weiß.

\footnotetext{
Literatur

1. Marx WM et al (2013) Ginger (Zingiber officinale) and chemotherapy-Induced nausea and vomiting: a systematic literature review. Nutr Rev 71(4):245-254

2. Memorial Sloan Kettering Cancer Center (MSKCC). About Herbs, Botanicals \& Other Products.Ginger (Stand 08/2014). https://www.mskcc.org/cancer care/ integrative-medicine/herbs/ginger

3. Qiu JX et al (2015) Estimation of the binding modes with important human cytochrome P450 enzymes, drug interaction potential, pharmacokinetics, and hepatotoxicity of ginger components using molecular docking, computational, and pharmacokinetic modeling studies. Drug Des Devel Ther 9:841-866
}

\title{
Kryokonservierung von Eizellen: befruchtet oder unbefruchtet?
}

\begin{abstract}
Hintergrund
Vor potenziell keimzellschädigenden Chemotherapien kann es bei Frauen sinnvoll sein, Eizellen zu entnehmen und zu konservieren, sofern die Familienplanung noch nicht abgeschlossen ist. Möglich ist dies mit befruchteten wie auch mit unbefruchteten Oozyten. Zur Frage, welche der beiden Methoden die größte Chance auf eine spätere erfolgreiche Schwangerschaft bietet, finden sich in der Literatur unterschiedliche Angaben. Die Kryokonservierung von befruchteten Eizellen wird bereits seit mehr als 25 Jahren erfolgreich weltweit angewendet. Sind die Zellen nach dem Auftauen intakt, hat die Implantation die gleichen Erfolgschancen wie bei frisch befruchteten Eizellen. Die Schwangerschaftsrate wird in Übersichtsarbeiten mit $25-59 \%$ beziffert [1,2].

Eine noch nicht so lange praktizierte Alternative ist das Einfrieren unbefruchteter Eizellen, das durch Fortschritte in der Konservierungstechnik heute ebenfalls als sicher und nicht mehr experimentell gelten kann [3]. Die Überlebens- und Befruchtungsraten entsprechen publizierten Daten zufolge etwa denjenigen frischer Eizellen [4,2] und betragen nach Schätzungen etwa 47\% bzw. 53\% [5]. Der Erfolg dieses Verfahrens wird allerdings sowohl durch die individuellen Voraussetzungen bei der Patientin als auch durch das Stimulationsverfahren, die Kryokonservierungstechnik und die später angewendete Befruchtungstechnik beeinflusst [4], sodass sich im individuellen Fall die Erfolgswahrscheinlichkeit nur schwer genau beziffern lässt. Mittlerweile sollen allerdings ähnliche Schwangerschaftsund Lebendgeburtsraten wie mit kryokonservierten befruchteten Eizellen erreichbar sein [2].
\end{abstract}

\section{Beide Verfahren vergleichbar erfolgreich: individuelle Beratung}

Die Kryokonservierungen befruchteter wie auch nichtbefruchteter Eizellen sind beides anerkannte und etablierte Methoden zum Erhalt der Fertilität bei Frauen, die sich einer Krebstherapie unterziehen müssen. Das Einfrieren befruchteter Eizellen setzt voraus, dass die Patientin einen festen Partner hat, dass die Krebserkrankung die erforderliche ovarielle Stimulation erlaubt und dass ausreichend Zeit dafür ist, also keine dringliche
Indikation zum unverzüglichen Beginn der Tumortherapie besteht. Dies muss mit der Patientin ausführlich besprochen werden [5]. Sind diese Voraussetzungen nicht gegeben, ist das Einfrieren nichtbefruchteter Eizellen eine erfolgversprechende Alternative. Auch ein Splitting kann ggf. sinnvoll sein, also die Kombination beider Strategien - für den Fall einer Trennung und späteren Kinderwunsches mit einem anderen Partner. Informationen und Beratung für Frauen und Männer bietet FertiPROTEKT, ein Netzwerk von Zentren und Ansprechpartnern für Fragen im Zusammenhang mit der Fertilitätserhaltung bei Krebstherapien [6].

\footnotetext{
Literatur

1. Kasum M et al (2014) Fertility after breast cancer treatment. Eur J Obstet Gynecol Reprod Biol 173:13-18

2. Rodriguez -Wallberg KA, Oktay K (2012) Options on fertility preservation in female cancer patients. Cancer Treat Rev 38(5):354-361

3. Practice Committees of American Society for Reproductive Medicine (ASMR) \& Society for Assisted Reproductive Technology (2013) Mature oocyte cryopreservation: a guideline. Fertil Steril 99(1):37-43

4. Cil AP, Seli E (2013) Current trends and progress in clinical applications of oocyte cryopreservation. Curr Opin Obstet Gynecol 25(3):247-254

5. Kovacs $P$ (2014) Fertility preservation in reproductive age women with cancer. J Obstet Gynaecol India 64(6):381-387

6. FertiPROTEC. http://www.fertiprotekt.de/
}

Korrespondenzadresse
A. Gaisser
Krebsinformationsdienst
Deutsches Krebsforschungszentrum
andrea.gaisser@dkfz-heidelberg.de

Auszug einer Veröffentlichung aus “ Der Onkologe” 10-2015 doi: 10.1007/s00761015-3034-x 